EPJ Web of Conferences 97,00031 (2015)

DOI: $10.1051 /$ epjconf/ 20159700031

(C) Owned by the authors, published by EDP Sciences, 2015

\title{
Baryon Resonances in the STAR Experiment at RHIC
}

\author{
Richard Witt ${ }^{1}$, a (for the STAR Collaboration) \\ ${ }^{1}$ United States Naval Academy, Annapolis, Maryland, 21402, USA
}

\begin{abstract}
We present measurements of mass, width, and yields of the $\Delta(1232)^{++}, \Sigma(1385)$ and $\Lambda(1520)$ from $p+p, d+A u$, and $A u+A u$ collisions at $\sqrt{s_{N N}}=200 \mathrm{GeV}$ in the STAR experiment at Brookhaven National Laboratory's Relativistic Heavy-Ion Collider (RHIC). These measurements are discussed in the context of re-scattering and regeneration. We also discuss preliminary measurements of the $\Xi(1530)$ and possibilities for upcoming measurements based on recently collected data.
\end{abstract}

\section{Introduction}

Hadronic resonances are readily produced in the hot dense environment of ultra-relativistic heavy-ion collisions. Resonance lifetimes vary by more than an order of magnitude, therefore some resonances are more likely to decay earlier during the evolution of the system than others. By taking advantage of the interplay of resonance daughter re-scattering and the production of resonances in the hadronic medium we may gain information about the partonic phase, the hadronic phase, and any difference in time between chemical freeze-out (fixing the particle ratios) and kinetic freeze-out (the time of last interaction) [1]. Furthermore, variations of the mean mass and/or width of resonance peaks have been suggested as a possible indicator of chiral symmetry restoration expected in the quark-gluon plasma phase [2]. Variation in the mean resonance mass for some meson resonances and the $\Delta^{++}$, and in the width of the $\Lambda^{*}$ has been observed in $p+p, d+\mathrm{Au}$, and $\mathrm{Au}+\mathrm{Au}[1,3-6]$.

\section{Masses and Widths}

In these proceedings the following decay channels were utilized for reconstructing the presented resonances; $\Delta^{++} \rightarrow p+\pi^{+}, \Sigma^{ \pm} \rightarrow \Lambda+\pi^{ \pm}, \Lambda(1520) \rightarrow p+K^{-}$, and $\Xi(1530)^{0} \rightarrow \Xi^{-}+\pi^{+}$. Charge conjugates of these decay channels were used for the antiparticles. Resonance masses and widths were obtained from the Breit-Wigner fits described below after subtraction of the combinatorial background by various techniques (also described below).

The mid-rapidity mass and width of the $\Delta^{++}$as functions of transverse momentum are shown in the left and right columns of Fig. 1 for minimally-biased $d+\mathrm{Au}$ and $p+p$ collisions respectively. They were obtained by fitting the $p \pi$ invariant mass distribution with a relativistic $p$-wave BreitWigner function [7] multiplied by a phase factor (see [5] for details). The $\Delta^{++}$mass is found to be significantly smaller than the standard Particle Data Group (PDG) value [8], while the width is within uncertainties of the expected value [8]. The main contributions to the systematic uncertainties were

ae-mail: witt@usna.edu

This is an Open Access article distributed under the terms of the Creative Commons Attribution License 4.0, which permits unrestricted use, distribution, and reproduction in any medium, provided the original work is properly cited. 

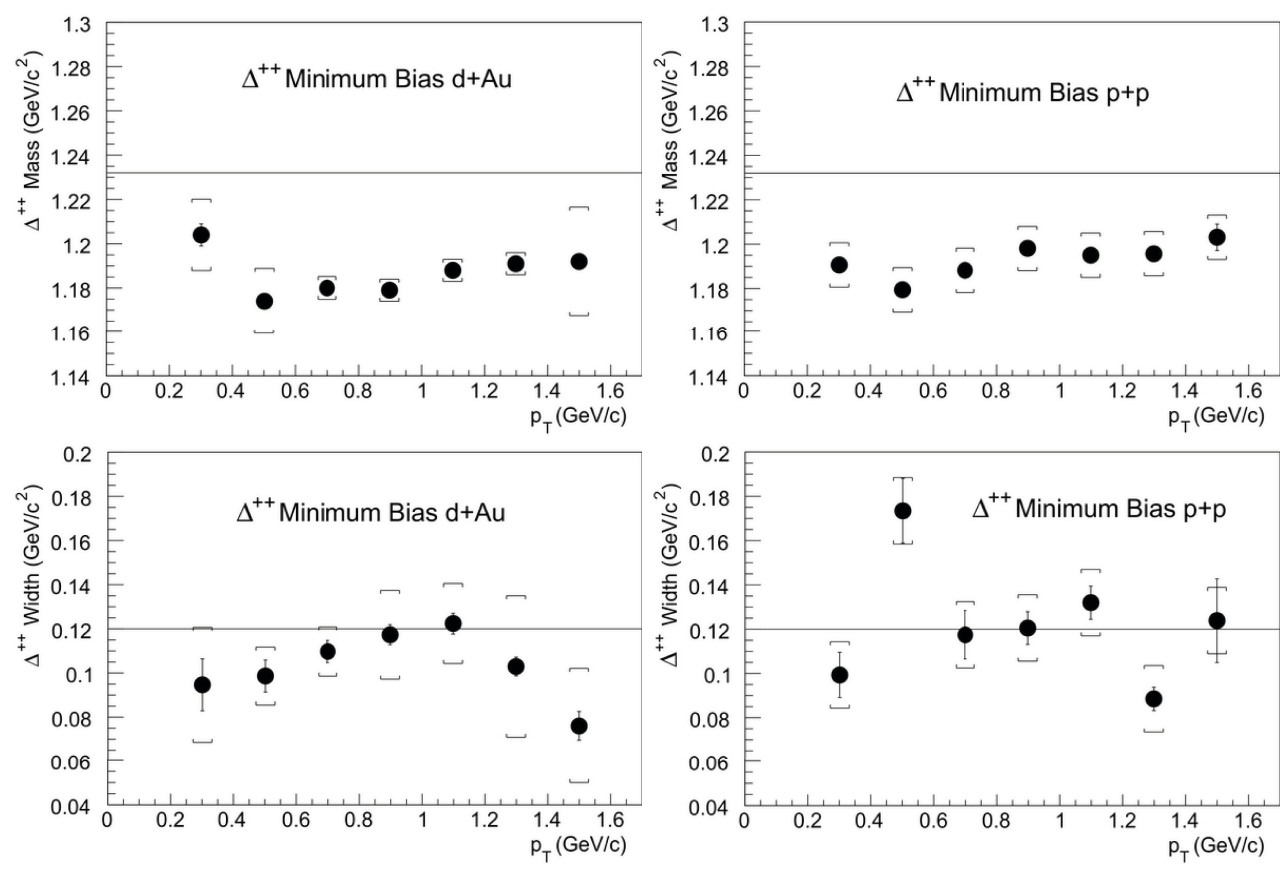

Figure 1. From [5], the $\Delta^{++}$mass (upper) and width (lower) from mid-rapidity $(|y|<0.5) d+\mathrm{Au}$ (left) and $p+p$ (right) collisions at $\sqrt{s_{N N}}=200 \mathrm{GeV}$. Solid lines indicate the mass and width from the Particle Data Group [8]. Systematic uncertainties are indicated by brackets.

found to be related to the function used to describe the residual background in the invariant mass distribution and to the slope parameter used in the phase space factor. In the $d+$ Au system the overall systematic uncertainty on the $\Delta^{++}$mass and width was found to be of the order of $20 \mathrm{MeV} / \mathrm{c}^{2}$ and $30 \mathrm{MeV} / c^{2}$, respectively. Systematic uncertainties in the $p+p$ system were determined in a similar manner and found to be of the order of $10 \mathrm{MeV} / c^{2}$ and $15 \mathrm{MeV} / c^{2}$ for the mass and width, respectively, of the $\Delta^{++}$.

Several explanations have been offered for the observed mass shift of the $\Delta^{++}$in $d+\mathrm{Au}$, including dynamical interactions with the surrounding matter, distortions of the phase space due to re-scattering of the daughters, and Bose-Einstein correlations with pions in the surrounding matter [3]. For the mass shift observed in $p+p$ it has been suggested that $\pi \pi$ re-scattering may be responsible since it does not require the presence of a medium [9].

Figure 2 shows the mid-rapidity mass and width of the $\Lambda(1520)$ and the mass of the $\Sigma(1385)$ from $d+$ Au collisions at $\sqrt{s_{N N}}=200 \mathrm{GeV}$ as functions of transverse momentum. The values were obtained fitting the invariant mass spectrum with a non-relativistic Breit-Wigner function plus either a Gaussian (in the $\Sigma(1385)$ case) or a linear function (in the $\Lambda(1520)$ case) to account for residual background. Residual background in the case of the $\Sigma(1385)$ comes mostly from the $\Xi$ and $\bar{\Xi}$, which have the same decay channels as the $\Sigma(1385)$ and $\bar{\Sigma}(1385)$. The $\Lambda(1520)$ mass is consistent with the expected value within uncertainties (indicated by brackets in Fig. 2). This figure would seem to indicate a significantly different width for the $\Lambda(1520)$ compared to the PDG value [8]. However, simulations that include both the detector resolution and the kinematic cuts indicate an expected width of 0.022 $\mathrm{GeV} / c^{2}$, which is consistent with our measurements within the quoted uncertainties. Identified sources 

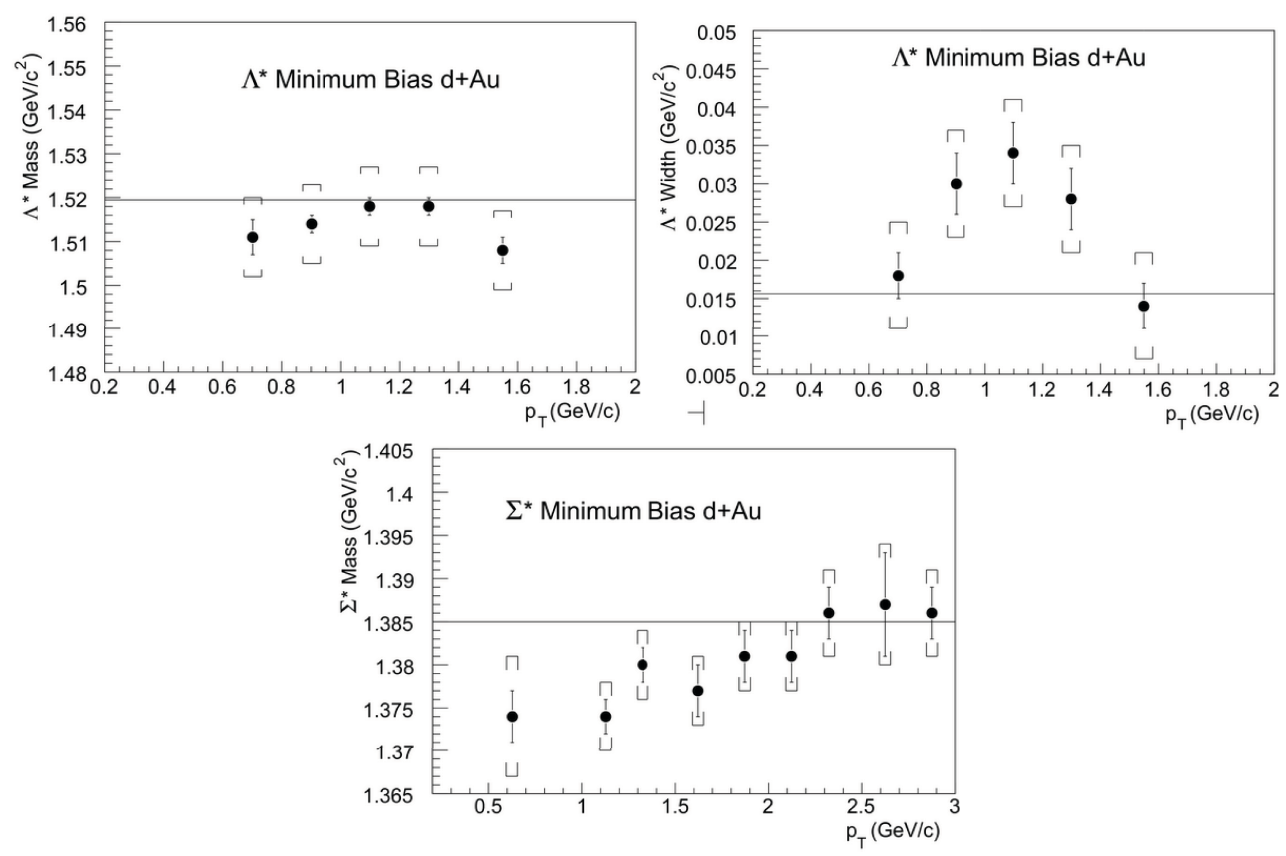

Figure 2. From [5], the $\Lambda(1520)$ mass (upper left) and width (upper right) and the $\Sigma$ (1385) mass (lower) from mid-rapidity $(|y|<0.5$ for $\Lambda(1520),|y|<0.75$ for $\Sigma(1385)) d+$ Au collisions at $\sqrt{s_{N N}}=200 \mathrm{GeV}$. Solid lines indicate the mass and width from the Particle Data Group [8]. Systematic uncertainties are indicated by brackets.

of systematic uncertainty include the choice of bin width, and of the range used for background normalization and fitting.

Both the mass and width of the $\Sigma(1385)$ were found to be consistent [5] with the values from the PDG [8]. However, if the width is held constant at the PDG value $\left(37.6 \pm 1.1 \mathrm{MeV} / c^{2}\right)$ and the mass remains a free parameter, a small deviation is observed in the low- $p_{T}$ region as seen in Fig. 2. Similar to the $\Lambda(1520)$, sources of systematic uncertainty for measurements on the $\Sigma(1385)$ include choice of bin sizes and fit range, event and track selection cuts, and normalization of the background distribution produced from the mixed-event technique [5]. The systematic uncertainties on the $\Sigma(1385)$ mass are shown as brackets in Fig. 2.

\section{Spectral Shapes}

Comparisons of spectral shapes may provide information on the relative contributions of hard and soft processes to particle production. Gluon saturation and the color glass condensate (CGC) suggest that identified hadron spectra may follow a generalized scaling law and fall onto an approximately universal curve versus transverse mass $\left(m_{T} \equiv \sqrt{p_{T}^{2}+m^{2}}\right)$ [10]. Saturation of the gluon density is not be expected in the $p+p$ system. Nevertheless, identified hadron spectra from $p+p$ collisions at ISR [11-13] and SPS [14] seem to follow an approximate scaling law. In Fig. 3 we present the transverse mass spectra of several identified baryons and mesons detected by the STAR detector in $p+p$ collisions [15] (left panel) and $d+\mathrm{Au}$ collisions [5] (right panel) at $\sqrt{s_{N N}}=200 \mathrm{GeV}$. In particular, for the $d+\mathrm{Au}$ collisions the spectra of several resonances $\left(\rho, K^{*}, \Delta, \Sigma^{*}\right.$, and $\left.\Lambda^{*}\right)$ are shown. The spectra in $p+p$ 

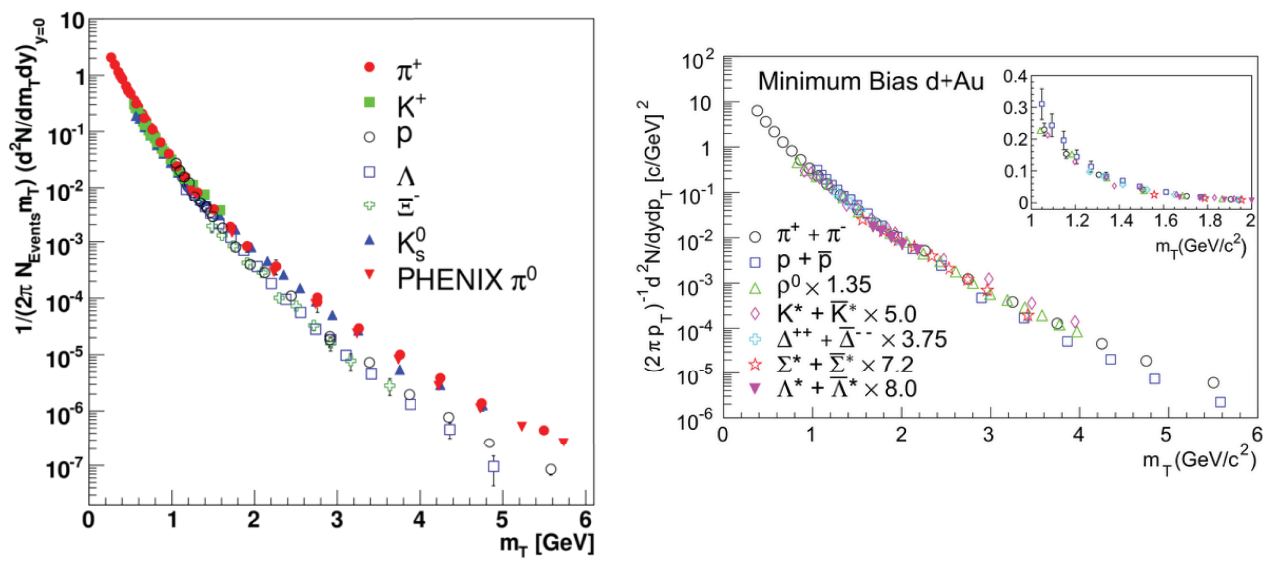

Figure 3. Mid-rapidity transverse mass spectra for several species of identified hadrons from minimum-bias $p+p$ collisions [15](left) and $d+\mathrm{Au}$ collisions [5](right) at $\sqrt{s_{N N}}=200 \mathrm{GeV}$. The spectra have been scaled by arbitrary factors that provide the best match at $m_{T} \approx 1 \mathrm{GeV}$. The scaling factors for the $d+\mathrm{Au}$ collisions are shown in the figure.

and $d+$ Au have been arbitrarily scaled to match at $m_{T} \approx 1 \mathrm{GeV}$. One aspect that immediately stands out in the $p+p$ data is the difference in spectral shape above $m_{T} \approx 2 \mathrm{GeV}$. Closer examination reveals the harder spectra to be exclusively those of the mesons. The proton and pion spectra from $d+\mathrm{Au}$ collisions still seem to diverge, although to a lesser degree, above $m_{T} \approx 4 \mathrm{GeV}$ but any such splitting among the resonances is mostly absent over the measured range. We echo the call in [5] for more theoretical work on the similarities of $p+p$ and $d+$ Au.

The average transverse momentum, $\left\langle p_{T}\right\rangle$, provides a means of partially characterizing the shape of the transverse momentum spectrum in a single number. Generally, for spectra of the same approximate shape, a larger $\left\langle p_{T}\right\rangle$ indicates a harder spectra. Figure 4 shows the $\left\langle p_{T}\right\rangle$ for various hadronic species, including resonances, from three different collision systems all at the same energy. A strong dependence on particle mass is immediately apparent and present in all three systems. A somewhat weak dependence on collision system could be inferred from the ordering of the points for each particle in $\left\langle p_{T}\right\rangle$, going from its lowest value in $p+p$ through $d+\mathrm{Au}$ to its highest value in central $\mathrm{Au}+\mathrm{Au}$ indicating harder spectra in the heavy-ion system compared to the $p+p$.

\section{Ratios}

The ratio of resonance yield to the yield of a stable particle with similar quark content can provide information on the dynamics of relativistic heavy-ion collisions $[1,3,4,16]$. Furthermore, a comparison of the resonance-to-stable ratio from $\mathrm{Au}+\mathrm{Au}$ collisions with the same ratio from $p+p$ collisions may provide some insight regarding the interplay of resonance decay daughter re-scattering and resonance (re-)generation in the hadronic phase of the collision. In Fig. 5, a "ratio of ratios" is shown, that is the ratio of the resonance-to-stable ratio from $d+\mathrm{Au}$ and from $\mathrm{Au}+\mathrm{Au}$ to that from $p+p$ [5].

As can be seen in the figure, the resonance-to-stable ratios of $\Lambda(1520) / \Lambda$ and $K(892) / K^{-}$are significantly smaller in $\mathrm{Au}+\mathrm{Au}$ than those from $p+p$. One explanation that has been provided is that a longer-lived hadronic phase in the $\mathrm{Au}+\mathrm{Au}$ system allows more re-scattering of the resonance decay daughters thereby decreasing the measured yield relative to that in $p+p$ [1]. Similarly, the relative 


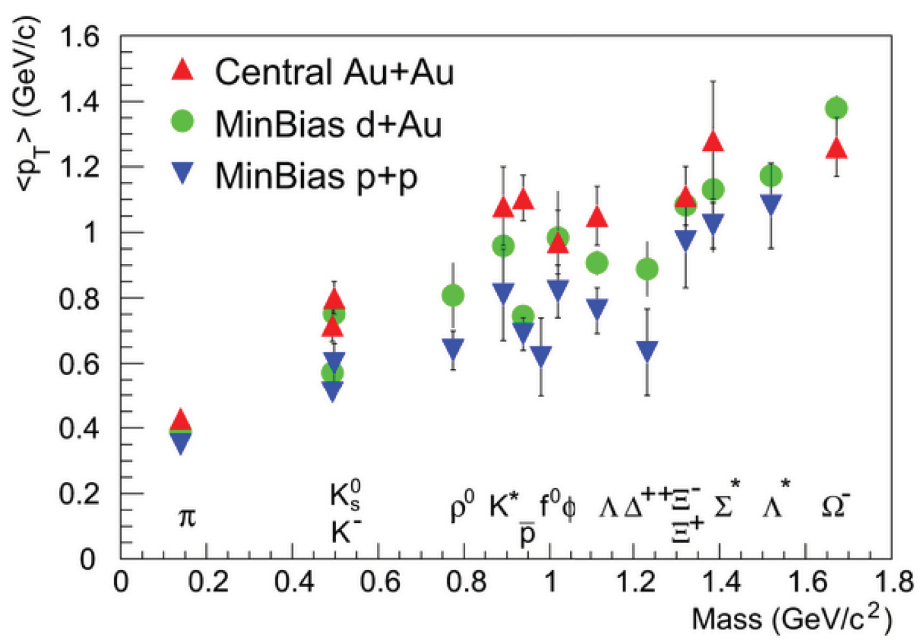

Figure 4. Mean transverse momentum for various hadrons from minimum-bias $p+p, d+\mathrm{Au}$, and central $\mathrm{Au}+\mathrm{Au}$ collisions at $\sqrt{s_{N N}}=200 \mathrm{GeV}$ [5]. The error bars indicate the combined statistical and systematic uncertainties, which were summed in quadrature.

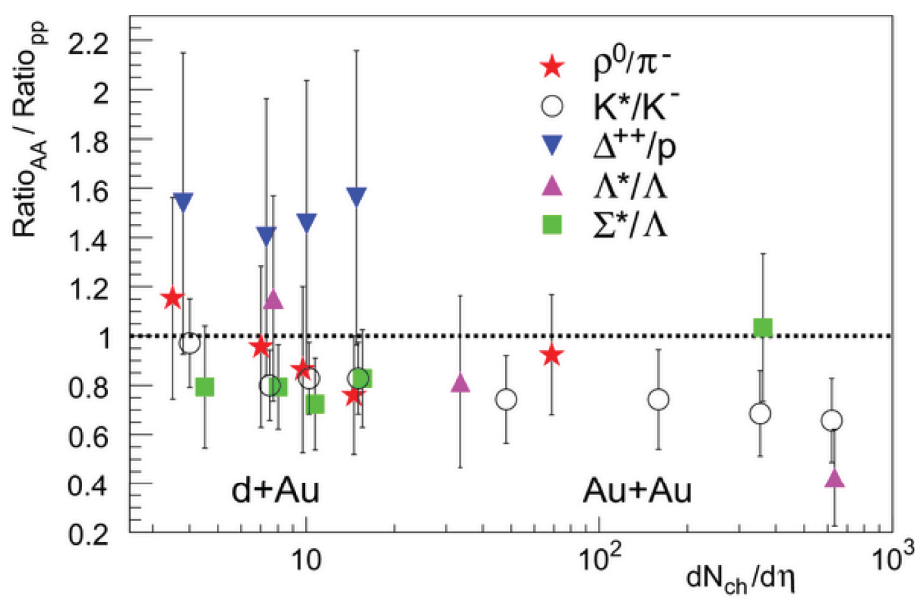

Figure 5. Ratio of resonance-to-stable ratio in $d+\mathrm{Au}$ and $\mathrm{Au}+\mathrm{Au}$ to that in $p+p$ for particles with similar quark content as a function of charged particle multiplicity at mid-rapidity [5]. The error bars indicate the combined statistical and systematic uncertainties, which were summed in quadrature.

agreement of the same ratios in $d+\mathrm{Au}$ and $p+p$ may indicate the absence of a long-lived hadronic phase in $d+$ Au collisions [5]. The $\Delta^{++} / p$ and $\Sigma(1385) / \Lambda$, as well as the $\rho^{0} / \pi^{-}$ratios show no dependence on multiplicity and collision system within uncertainties and may be insensitive to differences in the lifetime of the hadronic phase. This insensitivity might be expected if the $\Delta^{++}, \Sigma(1385)$, and $\rho^{0}$ regeneration cross sections are large [5]. 


\section{New Measurements on the $\Xi(1530)^{0}$}

One of the heaviest baryon resonances measured in heavy-ion collisions is the doubly-strange $\Xi(1530)^{0}$. Measurements of the $\Xi(1530)^{0}$ yields and spectra in $p+p$ and Au+Au collisions may provide further insight into the dynamics of heavy-ion collisions. The $\Xi(1530)^{0}$ is a narrow $(\Gamma \approx 9.1 \pm 0.5$ $\mathrm{MeV}$ ) resonance with a clean decay channel, $\Xi(1530) \rightarrow \Xi+\pi$, with a near $100 \%$ branching ratio making it easier to access experimentally than other resonances with many possible decay channels and/or large branching to neutral channels. Also, if the $\Xi+\pi$ cross section is small, modifications to the $\Xi(1530)^{0}$ yield should be dominated by re-scattering, allowing the $\Xi(1530)^{0}$ yield in heavy-ion collisions relative to that in $p+p$ (i.e. the nuclear modification factor $R_{A A}$ ) to serve as a measure of the degree of hadronic scattering occurring in the late stages of the collision evolution.
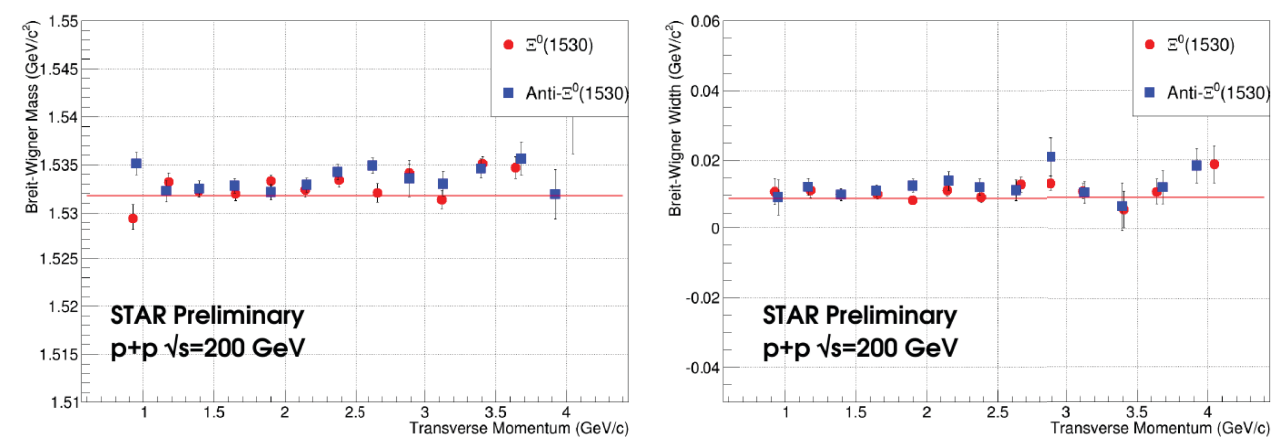

Figure 6. The mass (left) and width (right) of the $\Xi(1530)^{0}$ (filled circles) and anti- $\Xi(1530)^{0}$ (filled squares) from $p+p$ collisions in STAR at $\sqrt{s}=200 \mathrm{GeV}$. In each plot a horizontal line indicates the expected PDG value for mass and width. The error bars indicate the uncertainties on the Breit-Wigner fit parameters only.

We present in Fig. 6 measurements of the mass and width of the $\Xi(1530)^{0}$ (and its antiparticle) as functions of transverse momentum obtained from 151 million $p+p$ collisions at $\sqrt{s}=200 \mathrm{GeV}$ collected by STAR in 2009. The data shown were obtained by fitting a non-relativistic Breit-Wigner function to the invariant mass distribution of all $\Xi+\pi$ pairs after subtraction of the uncorrelated background estimated by the rotational method. The error bars in the figure indicate only the uncertainty on the Breit-Wigner fit parameter. An evaluation of the systematic uncertainty is underway. The current measurements are consistent with the PDG values, indicated by horizontal lines, within expected uncertainties across the entire measured range. The mean transverse momentum and resonance-tostable yield will be available once embedding corrections for efficiency and acceptance have been finalized. Measurement of the $p_{T}$ spectra in $p+p$ is crucial as a baseline in the nuclear modification factor $\left(R_{A A}\right)$. A high-statistics $p+p$ data set was collected by STAR during 2012 consisting of more than $1.7 \times 10^{9}$ events, which will allow extension of the spectra measurement to higher- $p_{T}$, a high$p_{T}$ azimuthal correlation measurement for both $\Xi(1530)^{0}$ and $\bar{\Xi}(1530)^{0}$ (similar [17]), and possibly spectral measurements in bins of multiplicity.

Lastly in Fig. 7, we present measurements of the mass and width of the $\Xi(1530)^{0}$ and $\bar{\Xi}(1530)^{0}$ in $10-20 \%, 30-40 \%$, and 70-80\% central Au+Au collisions at $\sqrt{s_{N N}}=200 \mathrm{GeV}$. As in Fig. 6, the mass and width are extracted by fitting the invariant mass distribution with a non-relativistic Breit-Wigner function. In this case a $2^{\text {nd }}$ order polynomial was added to describe the residual background after incoherent background subtraction estimated by the rotational method. Also as in Fig. 6, the error bars in the figure indicate only the uncertainty on the Breit-Wigner fit parameter. The mass and width 

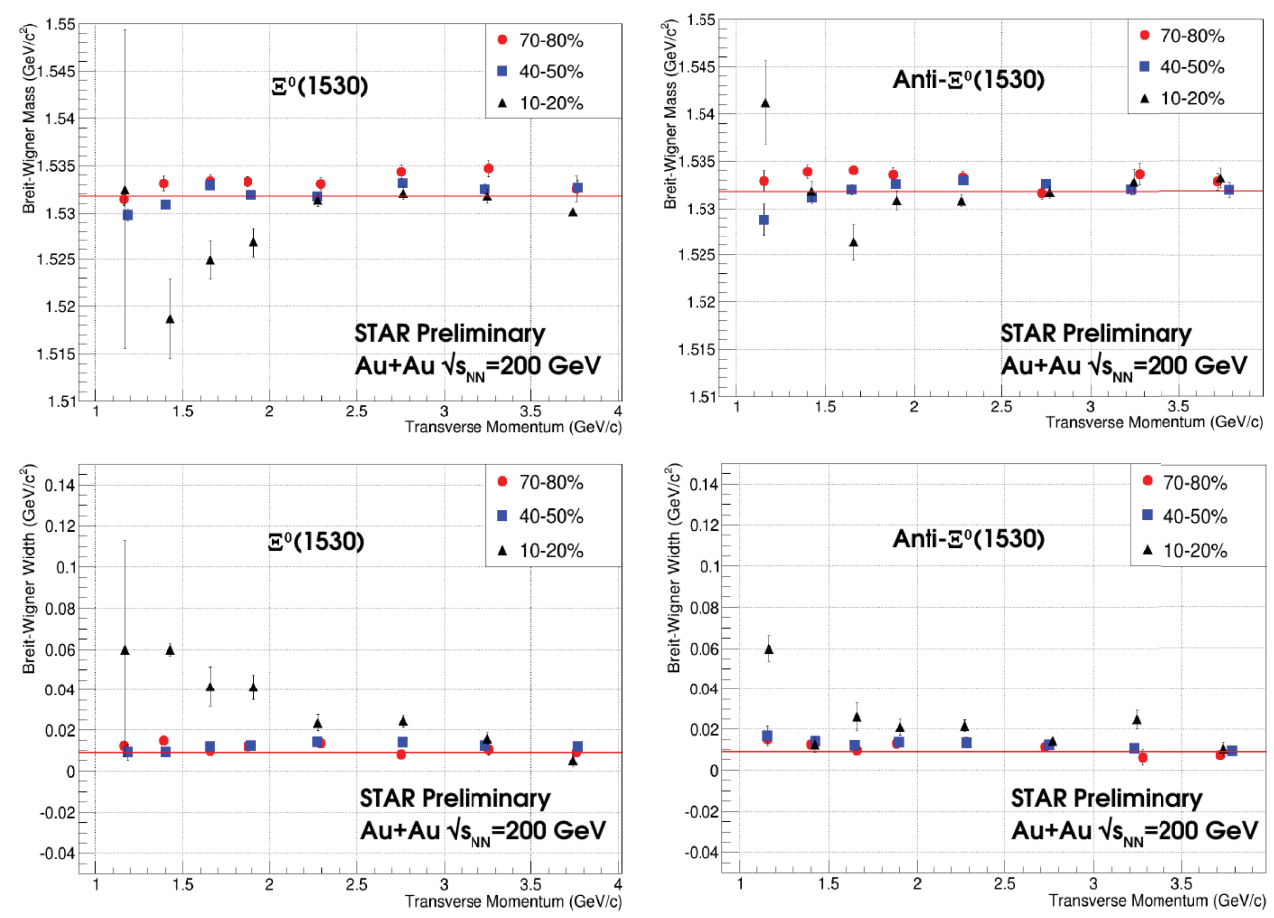

Figure 7. Measurements of the mass (upper) and width (lower) of the $\Xi(1530)^{0}$ (left column) and $\bar{\Xi}(1530)^{0}$ (right column) in $10-20 \%, 30-40 \%$, and $70-80 \%$ central $\mathrm{Au}+\mathrm{Au}$ collisions at $\sqrt{s_{N N}}=200 \mathrm{GeV}$. A horizontal line indicates the PDG mass and width in each plot.

for both the $\Xi(1530)^{0}$ and $\bar{\Xi}(1530)^{0}$ are consistent with the expected values from the PDG except the ones measured for $p_{T}<2 \mathrm{GeV} / \mathrm{c}$ in $10-20 \%$ central $\mathrm{Au}+\mathrm{Au}$. However, systematic uncertainties are still being evaluated so no strong conclusion can be drawn.

Furthermore, since the combinatorial background rises faster than the signal with collision centrality, analysis of high-statistics data from the 2014 run will be necessary to estimate the mass and width for the $0-5 \%$ and $5-10 \%$ centrality bins.

\section{Summary and Outlook}

We have discussed measurements of $\Delta^{++}, \Sigma(1385), \Lambda(1520)$, and $\Xi(1530)^{0}$ in collisions ranging from $p+p$ to Au+Au all at $\sqrt{s_{N N}}=200 \mathrm{GeV}$ in the STAR experiment at RHIC. The $\Delta^{++}$mass was found to be smaller than the PDG value by $\sim 40 \mathrm{MeV} / c^{2}$ in $p+p$ and $\sim 50 \mathrm{MeV} / c^{2}$ in $d+$ Au over the measured range. The $\Delta^{++}$width agrees with expectations in both collision systems. No such variation from the PDG values is found for the $\Sigma(1385)$ and $\Lambda(1520)$ in either system.

In $p+p$ collisions the transverse mass spectra of $\Delta^{++}, \Sigma(1385)$, and $\Lambda(1520)$ are found to follow a generalized scaling law in the range $1 \leq m_{T} \leq 2 \mathrm{GeV}$ though no such scaling is observed in $\mathrm{Au}+\mathrm{Au}$ collisions at $\sqrt{s_{N N}}=200$ and $62.4 \mathrm{GeV}[5,18]$. The generalized scaling of resonances in $d+\mathrm{Au}$ collisions at $\sqrt{s_{N N}}=200 \mathrm{GeV}$ is in contrast to the meson-baryon splitting that was observed among identified hadrons in $p+p$ collisions at the same collision energy. The $\left\langle p_{T}\right\rangle$ of $\Delta^{++}, \Sigma(1385)$, and $\Lambda(1520)$ is mass ordered and increases weakly with the size of the collision system. 
The suppression of the resonance-to-stable particle ratio measured in $\mathrm{Au}+\mathrm{Au}$ collisions for $\Lambda(1520) / \Lambda$ and $K(892) / K^{-}$with respect to the one in $p+p$ may indicate an extended lifetime of the source and therefore a dominance of re-scattering over resonance generation in the hadronic phase. No such suppression is observed for the $\Delta^{++} / p$ and $\Sigma(1385) / \Lambda$ ratios, possibly due to large regeneration cross sections. Similarly, little difference exists in the $\Lambda(1520) / \Lambda$ and $K(892) / K^{-}$ratios from $d+\mathrm{Au}$ and $p+p$ which may indicate the absence of an extended hadronic medium in the $d+$ Au system.

Finally, preliminary measurements with the STAR detector of the mass and width of $\Xi(1530)^{0}$ and $\bar{\Xi}(1530)^{0}$ in $p+p$ collisions and in $10-20 \%, 40-50 \%$, and $70-80 \%$ central Au+Au collisions at $\sqrt{s_{N N}}=200 \mathrm{GeV}$ have been presented. The masses and widths are found to be within uncertainties of values expected from the PDG above $p_{T}>2.0$. Systematic uncertainties are still being evaluated. We are hopeful that existing data from the 2012 and 2014 RHIC runs will allow in the near future, for baryon resonances, the measurement of mid-rapidity $p_{T}$ spectra and yields in the most central $\mathrm{Au}+\mathrm{Au}$ collisions, as well as azimuthal anisotropy $\left(v_{2}\right)$, high- $p_{T}$ correlations, nuclear modification factors, and possibly multiplicity-dependent studies in $p+p$.

\section{Acknowledgments}

We thank the RHIC Operations Group and RCF at BNL, the NERSC Center at LBNL, the KISTI Center in Korea, and the Open Science Grid consortium for providing resources and support. This work was supported in part by the Office of Nuclear Physics within the U.S. DOE Office of Science, the U.S. NSF, CNRS/IN2P3, FAPESP CNPq of Brazil, the Ministry of Education and Science of the Russian Federation, NNSFC, CAS, MoST and MoE of China, the Korean Research Foundation, GA and MSMT of the Czech Republic, FIAS of Germany, DAE, DST, and CSIR of India, the National Science Centre of Poland, National Research Foundation (NRF-2012004024), the Ministry of Science, Education and Sports of the Republic of Croatia, and RosAtom of Russia.

\section{References}

[1] B. I. Abelev et al., Phys. Rev. Lett. 97, 132301 (2006)

[2] R. D. Pisarski, Phys. Lett. B 110, 155 (1982)

[3] J. Adams et al., Phys. Rev. Lett. 92, 092301 (2004)

[4] J. Adams et al., Phys. Rev. C 71, 064902 (2005)

[5] B. I. Abelev et al., Phys. Rev. C 78, 044906 (2008)

[6] B. I. Abelev et al., Phys. Lett. B 673, 183 (2009)

[7] C. Adler et al., Phys. Rev. Lett. 89, 272302 (2002)

[8] K.A. Olive et al. (Particle Data Group), Chin. Phys. C 38, 090001 (2014)

[9] P. Fachini, R. S. Longacre, Z. Xu, and H. Zhang, J. Phys. G 34, 431 (2007)

[10] J. Schaffner-Bielich, D. Kharzeev, L. McLerran, and R. Venugopalan, Nucl. Phys. A705, 494 (2002)

[11] B. Alper et al., Nucl. Phys. B87, 19 (1975)

[12] K. Alpgard et al., Phys. Lett. B107, 310 (1981)

[13] G. Gatoff and C. Y. Wong, Phys. Rev. D 46, 997 (1992)

[14] J. Schaffner-Bielich et al., arXiv:nucl-th/0202054

[15] B. I. Abelev et al., Phys. Rev. C 75, 064901 (2007)

[16] C. Adler et al., Phys. Rev. C 66, 061901(R) (2002)

[17] M. M. Aggarwal et al., Phys. Rev. C 82, 24912 (2010)

[18] B. I. Abelev et al., Phys. Lett. B 655, 104 (2007) 